Revue internationale P.M.E.

Économie et gestion de la petite et moyenne entreprise

\title{
Repérer les compétences pour mieux comprendre le processus d'innovation
}

\section{Le cas d'une TPE touristique}

\section{Bénédicte Aldebert et Sabrina Loufrani-Fedida}

Volume 23, numéro 1, 2010

URI : https://id.erudit.org/iderudit/045744ar

DOI : https://doi.org/10.7202/045744ar

Aller au sommaire du numéro

Éditeur(s)

Presses de l'Université du Québec

ISSN

0776-5436 (imprimé)

1918-9699 (numérique)

Découvrir la revue

Citer cet article

Aldebert, B. \& Loufrani-Fedida, S. (2010). Repérer les compétences pour mieux comprendre le processus d'innovation : le cas d'une TPE touristique. Revue internationale P.M.E., 23(1), 33-61. https://doi.org/10.7202/045744ar
Résumé de l'article

Selon une enquête menée par Oséo en 2006, seule une frange réduite de PME et TPE serait vraiment organisée pour innover. Or, peu de travaux en sciences de gestion ont été menés pour comprendre comment ces PME et TPE arrivent à innover pour rester compétitives. Dès lors, l'objectif de notre travail est de combler partiellement ce manque. Plus précisément, nous cherchons à améliorer la compréhension du processus d'innovation dans les TPE, et ce, en mettant en évidence les compétences nécessaires à sa réalisation.

Pour ce faire, notre recherche repose sur l'étude longitudinale du processus d'innovation d'une TPE touristique, l'entreprise H2H (Hotel2Hotel).

L'analyse du cas, confrontée au modèle en chaîne de Kline et Rosenberg (1986) et à la théorie des ressources et compétences, permet, d'une part, de révéler six phases caractéristiques de l'innovation considérée $\mathrm{H} 2 \mathrm{H}$ et d'identifier cinq points de rupture marquant le passage entre les phases et, d'autre part, de repérer l'existence de compétences favorables au développement de l'innovation dans une TPE, à savoir les compétences technologiques, organisationnelles et environnementales. 


\title{
Repérer les compétences pour mieux comprendre le processus d'innovation Le cas d'une TPE touristique
}

Bénédicte ALDEBERT

Université de Toulouse

Sabrina LOUFRANI-FEDIDA

Université de Nice Sophia-Antipolis

\begin{abstract}
LES AUTEURES
BÉNÉDICTE ALDEBERT est maître de conférences en sciences de gestion à l'Université de Toulouse où elle enseigne la stratégie, le management des entreprises innovantes, la gestion de production et le diagnostic financier. Membre du Laboratoire d'études et de recherches appliquées en sciences sociales (LERASS EA 827), ses recherches s'articulent autour de trois thèmes principaux: le management stratégique des PME, l'innovation (création de valeur) et les TIC (conditions d'adoption) et privilégient une démarche multiméthodes (qualitative et quantitative). Adresse : Laboratoire LERASS - ORGANICOM, Université de Toulouse, 115 C, route de Narbonne, B.P. 67701, 31077 Toulouse, France. Courriel : <bene.aldebert@gmail.com>.

SABRINA LOUFRANI-FEDIDA est maître de conférences en sciences de gestion à l'Université de Nice Sophia-Antipolis où elle enseigne essentiellement la gestion des ressources humaines et le management de projet. Elle est responsable du master "communication organisationnelle " à l'IAE de Nice. Membre du Groupe de recherche en droit, économie et gestion (GREDEG, UMR CNRS 6227), ses recherches portent principalement sur le management des compétences et sur la dynamique des organisations, tout particulièrement dans des secteurs à fort potentiel technologique. Adresse: Laboratoire GREDEG UMR CNRS 6227, Université de Nice-Sophia Antipolis, 250, rue Albert-Einstein, 06560 Sophia-Antipolis, France. Courriel: <loufrani@gredeg.cnrs.fr>.
\end{abstract}

\section{RÉSUMÉ}

Selon une enquête menée par Oséo en 2006, seule une frange réduite de PME et TPE serait vraiment organisée pour innover. Or, peu de travaux en sciences de gestion ont été menés pour comprendre comment ces PME et TPE arrivent à 
innover pour rester compétitives. Dès lors, l'objectif de notre travail est de combler partiellement ce manque. Plus précisément, nous cherchons à améliorer la compréhension du processus d'innovation dans les TPE, et ce, en mettant en évidence les compétences nécessaires à sa réalisation.

Pour ce faire, notre recherche repose sur l'étude longitudinale du processus d'innovation d'une TPE touristique, l'entreprise H2H (Hotel2Hotel).

L'analyse du cas, confrontée au modèle en chaîne de Kline et Rosenberg (1986) et à la théorie des ressources et compétences, permet, d'une part, de révéler six phases caractéristiques de l'innovation considérée $\mathrm{H} 2 \mathrm{H}$ et d'identifier cinq points de rupture marquant le passage entre les phases et, d'autre part, de repérer l'existence de compétences favorables au développement de l'innovation dans une TPE, à savoir les compétences technologiques, organisationnelles et environnementales.

\begin{abstract}
According to a survey led by Oséo in 2005, only a small fringe of SME and SoHo would be really organized to innovate. However, few works in management were led to understand how these SME and SoHo manages to innovate to remain competitive. Therefore, the objective of our work is to fill partially this lack. Specifically, we seek to improve the understanding of the processes of innovation in home offices, and this, by bringing to light the skills necessary for its implementation.
\end{abstract}

To do this, our research is based on longitudinal study of the process of innovation of a very small tourist business: the $\mathrm{H} 2 \mathrm{H}$ (Hotel2Hotel).

The analysis of the case, confronted with the model in chain of Kline and Rosenberg (1986) and with the theory of the resources and the competencies, allows, on one hand, to reveal six characteristic phases of the innovation in $\mathrm{H} 2 \mathrm{H}$ and to identify five breaking points marking the transition between phases and, on the other hand, the existence of competencies favorable to the development of the innovation in a SoHo, namely the technological, organizational and environmental skills

\title{
RESUMEN
}

Según una investigación realizada por Oséo en 2005, sólo una pequeña parte de las muy pequeñas empresas y PyMEs sería bastante organizada para innovar. Pero, pocos estudios en Ciencias de la Gestión han intentado entender cómo estas empresas manejan para seguir siendo competitivas. Por esto, nuestro trabajo trata de llenar este vacío. En concreto, tratamos de mejorar la comprensión de los procesos de innovación de las muy pequeñas empresas, destacando las competencias necesarias para su aplicación.

Así, nuestra investigación se enfoque en el estudio longitudinal de procesos de innovación en un pequeño negocio de turismo, el $\mathrm{H} 2 \mathrm{H}$ (Hotel2Hotel).

El modelo de este caso, comparado a la cadena Kline y Rosenberg (1986) y a la teoría de recursos y competencias, nos permite, en primer lugar, revelar a las seis etapas de la innovación en $\mathrm{H} 2 \mathrm{H}$ y identificar a cinco puntos de ruptura que marcan 
la transición entre las fases y, en segundo lugar, permite identificar la existencia de competencias que puedan favorecer el desarrollo de la innovación en una muy pequeña empresa, o sea, los conocimientos tecnológicos, de organización y de medio ambiente.

\section{ZUSAMMENFASSUNG}

Laut einer Studie von Oséo (2006) sind nur ein Bruchteil der KMU und Mikrounternehmen organisiert, um Innovationen hervorzubringen. Und trotzdem gibt es nur wenige Studien, die aufzeigen, wie diese Kategorie von Unternehmen mit Innovationen wettbewerbsfähig bleiben können. Der vorliegende Bericht soll diese Lücke teilweise schliessen. Um dies zu erreichen, wird der Innovationsprozess in Mikrounternehmen und die dazu benötigten Kompetenzen genauer untersucht.

Die Untersuchung basiert auf einer Längsschnittstudie bei einem Kleinstunternehmen der Tourismusbranche ( $\mathrm{H} 2 \mathrm{H}$ - Hotel 2 Hotel).

Die Analyse des Falles anhand des Modells von Kline und Rosenberg (1986) und des ressourcen- und kompetenzbasierten Ansatzes bringt sechs Phasen und fünf Übergangspunkte des Innovationsprozesses bei $\mathrm{H} 2 \mathrm{H}$ hervor. Darüber hinaus werden die Kompetenzen (technologisch, organisatorisch und umweltbezogen), die für die Entwicklung von Innovationen in Mikrounternehmen förderlich sind, identifiziert.

\section{Introduction}

Reconnue comme source de croissance et de compétitivité, l'innovation, notamment appliquée aux petites entreprises, fait l'objet d'une attention croissante de la part des chercheurs en sciences de gestion depuis ces dernières années (Chanal, 2002; Fort, Rastoin et Temri, 2005; Ayerbe, 2006; Boldrini, 2008). Aussi, selon Bellon (1994), les petites et moyennes entreprises $(\mathrm{PME})^{1}$ semblent présenter des caractéristiques contradictoires à l'égard de l'innovation. D'un côté, par leur structure, elles ont une capacité de flexibilité interne et de réactivité plus importante que les grandes entreprises, ainsi qu'une souplesse dans leur communication interne et une rapidité des prises de décisions. D’un autre côté, elles sont supposées ne pas posséder

1. Une TPE (très petite entreprise) est considérée comme une entreprise dont l'effectif est compris entre 0 et 19 salariés. Les TPE sont une sous-catégorie de PME (avec des similitudes fortes et des différences) et nous considérons dès lors que la spécificité des TPE réside davantage dans le fonctionnement et les comportements stratégiques que dans leur taille exclusivement. Cet article ne vise pas à débattre des différences entre PME et TPE, mais bien à analyser les spécificités du processus d'innovation d'une entreprise de petite taille. Aussi, au cours de l'article, les appellations PME et TPE seront toutes deux employées, dans la mesure où l'objet de notre terrain d'étude porte sur le processus d'innovation d'une TPE qui fédère un réseau de PME. 
les ressources et compétences internes, surtout humaines et financières, nécessaires pour les projets d'innovation. L'enquête réalisée par Oséo ${ }^{2}$ en 2005 auprès de 1000 PME explique ce point par le fait que seule une frange réduite de PME serait vraiment organisée pour innover.

Partant de ces constats, l'objectif de cet article est d'améliorer la compréhension du processus d'innovation dans les PME et, plus particulièrement les TPE, et ce, en mettant en évidence les compétences nécessaires à sa réalisation. En d'autres termes, nous cherchons à apporter des éléments de réponse aux questions suivantes: Comment se réalise un processus d'innovation dans une TPE? Quelles sont les compétences nécessaires pour sa réussite?

Pour ce faire, d'un point de vue théorique, nous mobilisons le modèle en chaîne de Kline et Rosenberg (1986) et une conceptualisation de l'entreprise puisée dans la théorie des ressources et compétences (Wernerfelt, 1984; Prahalad et Hamel, 1990; Barney, 1991). D’un point de vue méthodologique, notre recherche repose sur l'étude longitudinale du processus d'innovation d'une TPE touristique, l'entreprise $\mathrm{H} 2 \mathrm{H}$ (Hotel2Hotel). En effet, observant que la plupart des travaux de recherche sur l'innovation se concentre sur les ressources et compétences liées à la $\mathrm{R}-\mathrm{D}$, nous avons choisi de mener une étude empirique sur un secteur reconnu comme faiblement intensif en R-D et pourtant considéré comme dynamique en termes d'innovation: l'industrie du tourisme (Decelle et Tassin, 2005). En particulier, grâce aux technologies de l'information et de la communication (TIC), l'entreprise $\mathrm{H} 2 \mathrm{H}$ a créé un service de réservation entre hôteliers, tout à fait original pour le marché. Ce cas est révélateur au sens de Yin (1994), dans la mesure où il montre comment l'innovation $\mathrm{H} 2 \mathrm{H}$ a su bénéficier de l'aide d'autres structures pour surmonter son manque de ressources et acquérir les compétences qui lui faisaient défaut.

Cet article comprend quatre parties. Dans une première partie, nous présentons le cadre théorique mobilisé dans notre recherche. Dans une deuxième partie, nous exposons l'étude empirique. La troisième partie détaille, quant à elle, les analyses empiriques et les principaux résultats de notre recherche. Enfin, la quatrième partie propose une discussion de ces résultats au regard de la littérature existante.

2. Établissement public de l'État, l'Oséo a pour mission de financer et d'accompagner les PME en partenariat avec les banques et les organismes de capital investissement. 


\section{Cadre théorique}

L'objectif de cette première partie est double. Il s'agit, dans un premier temps, de présenter une caractérisation des TPE innovantes et, dans un second temps, de poser les deux modèles théoriques mobilisés pour l'analyse de notre étude empirique: le modèle en chaîne de Kline et Rosenberg (1986) et la théorie des ressources et des compétences.

\subsection{Vers une caractérisation des TPE innovantes en termes de proximité}

Nous souhaitons ici porter un regard sur les caractéristiques des TPE innovantes. Pour ce faire, nous avons ancré nos réflexions sur les travaux de Torrès $(1997,2004,2007)$ et Jaouen et Torrès (2008). Dans un premier temps, Torrès (1997) soutient qu'une entreprise de petite taille peut ne pas correspondre à la conception classique de la PME telle qu'elle est décrite dans la littérature, dans la mesure où la thèse de la spécificité de la PME ne doit pas être prise comme un paradigme, mais comme une simple hypothèse de recherche contestable. Les PME et TPE ne sont pas une grande entreprise miniature. En particulier, en matière d'innovation, outre le critère de taille, les PME ont des processus et des procédures de prises de décisions singuliers (Torrès, 1997). Dans un second temps, et c'est ce point qui a le plus retenu notre attention $^{3}$, Torrès $(2004,2007)$ ainsi que Jaouen et Torrès (2008) définissent la spécificité des PME et TPE comme une recherche protéiforme de la proximité, que ce soit au sein de l'entreprise comme dans ses relations avec ses clients, fournisseurs et autres partenaires. Cette approche en termes de proximité nous conduit à dégager un profil large des TPE innovantes à partir de cinq caractéristiques : hiérarchique, fonctionnelle, système d'information, temporelle et spatiale.

Une proximité hiérarchique. Les prises de décision de la TPE sont très centralisées (Marchesnay, 1997) et le management souvent concentré exclusivement sur une personne: le propriétaire-dirigeant. Cette centralisation du pouvoir du dirigeant est effective grâce à une forte proximité du dirigeant et de ses employés qui partagent le même lieu et les mêmes conditions de travail. De fait, la ligne hiérarchique est courte et les décisions sont prises par un petit nombre d'intervenants. Il apparaît également que la capacité du dirigeant à s'approprier et à intégrer de nouveaux savoirs est fondamentale dans le processus d'innovation de la TPE. Cette proximité hiérarchique

3. Les auteures tiennent à remercier un des évaluateurs pour la mise en avant de cette remarque pour la caractérisation des TPE innovantes en termes de proximité, lors de la révision du texte. 
s'accompagne d'une multiplication des contacts personnels et directs (Torrès, 2004). Ces échanges, pour la plupart non standardisés, favorisent la créativité au sein de l'entreprise.

Une proximité fonctionnelle. La division du travail n'est pas très intensive au sein des TPE. Elles sont souvent spécialisées autour d'un métier et ne sont pas divisées en services relativement cloisonnés et identifiés. Les TPE sont souvent reconnaissables à un métier particulier ou à un savoir-faire central qui conditionne les stratégies possibles (Bréchet, 1990). De nombreuses fonctions (production, marketing, finance, ressources humaines) sont réalisées par le dirigeant ou par un ensemble d'acteurs polyvalents qui mènent des tâches de nature très variées. Il est alors difficile de différencier les tâches. Cette proximité fonctionnelle conduit, selon Torrès (2004), au fait que les acteurs sont en permanence en contact et au courant des problèmes auxquels font face les autres fonctions. Cette proximité permet d'interconnecter l'ensemble des personnels et favorise l'initiative et la résolution de problèmes courants propices à l'innovation. Cette dernière sollicitant quasiment l'ensemble des fonctions d'une entreprise, il semble que la configuration structurelle des TPE soit proche de celle définie par Mintzberg (1982): l'adhocratie. Cette dernière caractérise d'ailleurs la plupart des entreprises innovantes, dans la mesure où son but est la détection de nouvelles opportunités, la résolution de problèmes et l'obtention de résultats en incitant la motivation individuelle.

Un système d'information de proximité. Au niveau interne, le traitement de l'information favorisé par les dirigeants est rapide et opérationnel. Ils privilégient des médias informels, des outils simples et des informations verbales, du fait de la proximité physique des acteurs. D'un point de vue externe, Torrès (2004) considère que le système d'information (SI) de la TPE est simple car le marché n'est pas de grande envergure et relativement proche. En effet, le dirigeant connaît souvent, personnellement, ses clients et ses fournisseurs. Les TPE peuvent répondre rapidement aux changements locaux. D'ailleurs, Julien (1996) indique que «plus les PME sont reliées à des réseaux informationnels riches, plus elles peuvent saisir les opportunités avant les autres tout en s'assurant d'informations essentielles pour compléter leurs ressources effectives ou potentielles» (p. 1140).

Une proximité temporelle. La stratégie de la TPE est implicite et de court terme. Les TPE sont souvent en situation d'urgence, leurs prises de décisions sont souvent rapides et opérationnelles et elles ont, pour cela, une grande capacité d'improvisation. Elles réagissent très rapidement aux contraintes et opportunités du présent. Compte tenu du fait qu'elles ont une ligne hiérarchique courte, elles sont flexibles et peuvent rapidement, dans le temps, ajuster leur stratégie et implanter leurs décisions en réponse au marché. 
Une proximité spatiale. Selon Torrès (2004, 2007), l'environnement que considère la TPE est un environnement de proximité. Pour l'auteur, les TPE présentent une insertion territoriale forte, leur espace marchand étant restreint. Il précise d'ailleurs que cet enracinement et les liens de proximité tissés avec l'environnement local permettent aux TPE d'être souvent incubatrices d'idées nouvelles, grâce à une connaissance précise des besoins de leurs clients.

Après avoir rappelé les caractéristiques des TPE innovantes en termes de proximité, nous proposons à présent de nous intéresser au processus d'innovation lui-même.

\subsection{Le processus d'innovation de Kline et Rosenberg (1986): remise en cause de la place de la recherche et valorisation du rôle du marché}

Dans la littérature et à l'instar des travaux de Loilier et Tellier (1999), nous avons repéré deux grands modèles d'analyse du processus d'innovation: les «linéaires» et les «interactifs». Les modèles linéaires se sont construits sur une structure unidirectionnelle, comprenant le passage progressif d'une étape du processus à une autre. L'innovation est considérée ici comme l'enchaînement linéaire et séquentiel de plusieurs étapes correspondant chacune à une phase indispensable à la finalisation du projet (recherche fondamentale $\rightarrow$ recherche appliquée $\rightarrow$ invention $\rightarrow$ développement $\rightarrow$ innovation $\rightarrow$ production $\rightarrow$ marché $)^{4}$.

Parallèlement aux modèles linéaires, des modèles qualifiés par Loilier et Tellier (1999) «d'interactifs» se sont développés. Ces modèles non linéaires réfutent le principe de séquentialité et mettent l'accent sur les phénomènes d'interaction et de rétroaction (feedbacks) qui ont lieu tout au long du processus d'innovation. Nous avons recensé deux principaux modèles non linéaires. Le modèle de Kline et Rosenberg (1986) introduit des interactions entre chacune des étapes. Le modèle tourbillonnaire d'Akrich, Callon et Latour (1988) définit l'innovation à travers les interactions entre les différents acteurs. Parmi ces deux modèles majeurs interactifs, nous avons retenu le modèle dit de la «chaîne interconnectée» (The Chain-Linked Model) de Kline et Rosenberg (1986), comme représenté dans le schéma suivant ( $c f$. schéma 1). Les raisons justifiant ce choix seront exposées à la suite de ce dernier.

4. Dans le modèle linéaire ou séquentiel, le processus d'innovation est souvent illustré par la métaphore sportive de la course de relais, car une étape ne peut commencer que si celle qui la précède est terminée. 
SCHÉMA 1

Le modèle en «chaîne interconnectée»*

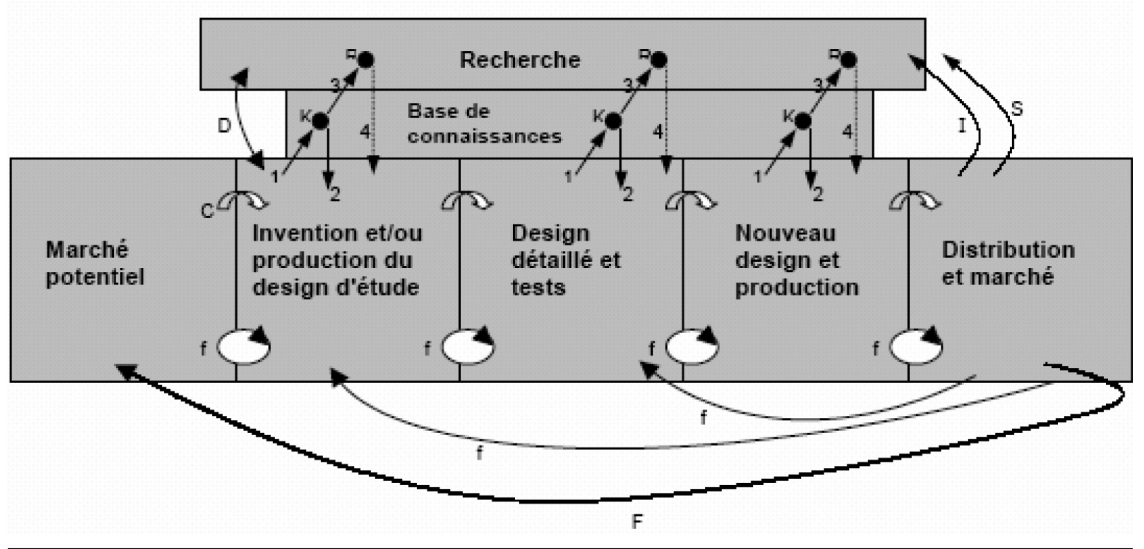

* Le processus d'innovation de Kline et Rosenberg (1986) se présente comme un ensemble de cinq sentiers ou chemins. Le premier est la succession des étapes d'invention, de développement, de production et de mise sur le marché, représentée par les flèches $C$ dans la chaîne centrale. L'identification d'un marché potentiel, sans lequel l'innovation ne pourrait être initiée, est le point de départ de ce sentier. Le deuxième correspond aux liens de retour (feedbacks) entre deux étapes de la chaîne centrale, étapes concomitantes, étapes plus éloignées (flèches F) et les étapes aux extrémités de la chaîne (flèches $F$ ), le troisième aux liens entre la science et les étapes de conception de la chaîne de l'innovation, passant d'abord par les connaissances (flèches K); si ces dernières n'apportent pas de solutions, alors la recherche est engagée (flèche $\mathrm{R}$ ). Le quatrième est le lien direct entre la science et l'invention (flèche $\mathrm{D}$ ) et le cinquième, le lien direct entre la recherche et les produits de l'innovation (flèche $\mathrm{S}$ ).

Source: Kline et Rosenberg (1986, p. 286).

L'un des intérêts du modèle de Kline et Rosenberg (1986) est l'introduction des interactions entre chacune des étapes du processus d'innovation. Dans le schéma ci-avant, la chaîne centrale de l'innovation est composée de cinq parties, dont trois concernent la conception et deux, le marché. Deux autres éléments extérieurs à la chaîne centrale, mais en lien avec elle, s'intéressent à la science (recherche et connaissances).

Si les auteurs excentrent la partie recherche, c'est qu'à partir de nombreux exemples historiques, ils ont constaté que la recherche n'est pas systématiquement utilisée pour innover. Selon eux, elle forme un goulet en ce qu'elle garantit soit le succès, soit l'échec de l'innovation. Kline et Rosenberg (1986) affirment également que la recherche n'est mobilisée que lorsque les connaissances existantes ne sont pas suffisantes pour résoudre les problèmes 
auxquels ils doivent faire face. Les différentes étapes de ce modèle sont liées les unes aux autres et autorisent des retours en arrière. Ainsi, le processus d'innovation est complexe et résulte d'opérations d'essai-erreur.

Dans le modèle, la conception ou design de l'innovation est une étape centrale: «la conception est essentielle pour initier l'innovation technique, et les reconceptions sont essentielles pour aboutir au succès. Le processus central de l'innovation n'est pas la science mais la conception » (Kline et Rosenberg, 1986, p. 286). De plus, ce modèle se base sur une étroite coordination du marché et de la technologie, visant à offrir les produits les plus adéquats aux besoins des utilisateurs. C'est en cela que la contribution de Kline et Rosenberg (1986) est intéressante, dans la mesure où elle place le marché et la conception au centre du processus d'innovation et la science (composée de la recherche et des connaissances existantes) à l'extérieur de la chaîne centrale.

Ainsi, ce modèle montre que la recherche peut être mobilisée pour résoudre des problèmes qui se posent tout au long du processus d'innovation, que le marché joue un rôle aussi important que la technologie dans le processus d'innovation et que le succès de l'innovation tient à la coordination des activités. Enfin, Kline et Rosenberg (1986) justifient la mobilisation de la recherche lorsque les connaissances existantes sont limitées. Ils ne précisent cependant pas les facteurs explicatifs du passage d'une phase à une autre et pourquoi les entreprises cherchent souvent, dans leur environnement et non seulement dans leur base interne de connaissances, les ressources et compétences qui leur manquent. Devant ce constat, nous proposons de mobiliser le cadre théorique du modèle des ressources et compétences, pour améliorer notre compréhension du processus d'innovation dans les PME.

\subsection{Les apports de la théorie des ressources et compétences au processus d'innovation dans les TPE}

Wernerfelt (1984), l'un des fondateurs de la théorie des ressources, traduit de l'anglais «resource-based view» $(\mathrm{RBV})$, définit une ressource comme un actif tangible ou intangible, ou comme une force ou faiblesse, rattachée de manière semi-permanente à la firme. D'une manière générale, la distinction, très souvent utilisée dans la littérature, consiste à séparer les ressources tangibles (humaines, financières ou matérielles) des ressources intangibles (information, réputation, savoir-faire et connaissances) au sein de l'organisation (Nanda, 1996). Certaines ressources d'une entreprise peuvent se révéler intransférables à d'autres organisations et lui conférer, par là-même, un avantage concurrentiel (Barney, 1991). 
À partir des travaux précurseurs de la RBV (Wernerfelt, 1984; Barney, 1991), nous pourrions croire que l'unité d'analyse fondamentale, voire unique, est représentée par les ressources. Toutefois, une attention particulière est également accordée aux compétences de l'entreprise (Prahalad et Hamel, 1990; Grant, 1991). D'une manière générale, la théorie des ressources présente les compétences de l'entreprise comme la résultante de la combinaison coordonnée et valorisante des différentes ressources. Cependant, pour bien appréhender les concepts de cette théorie, il est important d'établir une distinction nette entre ressources et compétences, c'est-à-dire de différencier ce qu'une entreprise possède (les ressources) de ce qu'elle sait faire (les compétences). Par conséquent, la principale distinction entre une ressource et une compétence est qu'une compétence doit être mise en œuvre pour exister et perdurer.

Certes, le portefeuille de ressources et compétences (R-C) détenu par l'entreprise est important, mais la manière dont elle les combine et les mobilise importe au moins tout autant. C'est ainsi que parallèlement à la reconnaissance du rôle des R-C, les années 1990 ont vu apparaître une prise de conscience accrue de l'importance des capacités relatives des firmes à utiliser leurs R-C actuelles, à créer de nouvelles R-C et à concevoir de nouvelles manières d'utiliser ces deux types de $\mathrm{R}-\mathrm{C}$ (actuelles et nouvelles). Des recherches se sont alors intéressées à la notion de capacité dynamique. Notamment, Teece, Pisano et Shuen (1997) définissent cette dernière comme «l'aptitude d'une firme à intégrer, construire et reconfigurer ses compétences internes et externes pour faire face aux changements rapides de l'environnement» (p. 516). Dès lors, l'avantage concurrentiel d'une entreprise se trouve dans ses processus organisationnels et managériaux («coordination et intégration »; «reconfiguration et transformation»), qui dépendent de ses actuelles positions en ressources.

Dans le cadre de notre recherche, la mobilisation de la théorie des R-C est intéressante dans la mesure où elle complète les travaux de Kline et Rosenberg (1986) en apportant une explication du passage d'une phase à l'autre du processus d'innovation. En effet, une des caractéristiques manifestes des TPE est leur manque de R-C. Cette insuffisance vaut pour tout type de ressource: humaine, financière, matérielle, technologique, informationnelle, etc. En particulier, selon Bellon (1994), les PME sont supposées ne pas posséder les R-C internes, surtout humaines et financières, nécessaires pour les projets d'innovation. Pourtant, le manque de ressources humaines est particulièrement pénalisant puisqu'elles sont reconnues comme essentielles à l'innovation (François et al., 1999). L'insuffisance financière, de son côté, compromet l'investissement. En effet, les TPE manquent souvent de fonds propres pour mener des projets d'innovation. Leur capacité à proposer 
des offres nouvelles s'en trouve nécessairement diminuée. Pour faire face à cette lacune, les TPE sont incitées à acquérir de nouvelles compétences. Il existe, à ce jour, plusieurs manières pour une organisation d'accéder à des compétences qu'elle ne possède pas. Selon Tarondeau (2002), au-delà des compétences innées qui proviennent des fondateurs des organisations et des pratiques institutionnalisées adoptées dès la naissance de l'organisation, les compétences de l'entreprise peuvent être transférées ou imitées en externe, acquises ou développées en interne. Pour faire le lien avec le modèle de Kline et Rosenberg (1986), les compétences développées en externe correspondraient à l'exploration de nouvelles connaissances via la «recherche » et l'apprentissage interne des compétences se traduirait par l'exploitation des connaissances existantes.

Pour résumer et conclure notre analyse théorique, nous pouvons affirmer qu'en matière d'innovation, la TPE se trouve devant une articulation délicate. D'un côté, elle dispose de caractéristiques reconnues comme importantes pour rester compétitive par rapport aux grandes entreprises: flexibilité interne, réactivité, souplesse dans la communication interne et rapidité des prises de décision. Toutefois, de l'autre côté, elle présente des carences en termes de ressources et compétences nécessaires pour les projets d'innovation. Aussi, nous avons trouvé peu d'études cherchant à explorer cette articulation. Pour combler partiellement ce vide, nous proposons d'améliorer la compréhension du processus d'innovation dans les TPE, et ce, en repérant les compétences nécessaires à sa réalisation. En d'autres termes, nous cherchons à apporter des éléments de réponse aux questions suivantes: Comment se réalise un processus d'innovation dans une TPE? Quelles sont les compétences nécessaires pour sa réussite?

\section{2. Étude empirique}

Notre recherche repose sur une démarche qualitative, centrée sur une étude longitudinale du processus d'innovation d'une TPE touristique, l'entreprise $\mathrm{H} 2 \mathrm{H}$ (Hotel2Hotel). La démarche qualitative a été privilégiée car elle autorisait la prise en compte de la dimension temporelle d'un processus dont l'importance dans la compréhension des phénomènes organisationnels, notamment ceux relatifs à l'innovation, est reconnue entre autres par Van de Ven et Poole (1990).

\subsection{Recueil et analyse des données}

La principale source de données a été l'entretien individuel. Vingt-cinq entretiens semi-directifs, d'une durée moyenne de une heure trente, ont 
été réalisés, à l'aide d'un guide d'entretien, auprès des dirigeants de l'entreprise. Parallèlement à cette investigation, une analyse documentaire nous a permis de compléter les informations issues des entretiens et de comprendre l'évolution de l'entreprise avant notre arrivée (manuel de fonctionnement du logiciel, revue de presse, documents administratifs et facturation, statuts juridiques, etc.). Enfin, une observation participante a été menée, en prenant part à des activités exceptionnelles et ponctuelles de l'entreprise (réalisation du plan d'affaires ou encore réponse à des appels à projets). Nous avons retranscrit nos observations dans un journal de recherche. Au total, le recueil intensif des données a duré six mois, d'octobre 2004 à mars 2005, soit 32 journées de présence sur le terrain.

Quant à l'analyse des données, elle repose sur une analyse processuelle par les phases. Cette dernière nous a semblé être la démarche la plus adéquate pour décrire et comprendre le processus d'innovation $\mathrm{H} 2 \mathrm{H}$, à travers la description des différentes phases qui ont marqué son histoire, depuis l'émergence de l'idée en 2001 jusqu'à sa commercialisation en 2005. L'analyse du processus vise ainsi à mettre en évidence le déroulement du phénomène étudié dans le temps. La difficulté majeure d'une analyse processuelle par les phases est de savoir comment décomposer le processus. Pour cela, Van de Ven et Poole (1990) préconisent d'identifier des phases et des points de rupture du phénomène étudié. Les différentes phases consistent en des activités plus ou moins unifiées et cohérentes au cours du processus, alors que les points de rupture constituent les transitions et les points de passage entre les phases (Van de Ven et Poole, 1990). Plus précisément, les points de rupture affectent les actions suivantes et permettent ainsi de rendre compte de l'évolution du phénomène. Selon Poole (1983), il existe trois types de points de rupture: les points de rupture normaux qui sont le résultat d'une procédure normale; les délais qui traduisent une période où le phénomène observé est arrêté ou en suspens; ou une interruption qui caractérise un conflit interne ou un résultat inattendu.

En analysant les récits des dirigeants de $\mathrm{H} 2 \mathrm{H}$, nous avons pu relever six phases homogènes et cinq points de rupture dans le processus d'innovation $\mathrm{H} 2 \mathrm{H}$. Chaque phase représente une forme de cycle de vie d'activités avec leur émergence, leur développement et leur déclin. Le déclin de chaque phase est marqué par un obstacle, une limite en termes de compétences auxquels les associés sont confrontés. Cela les conduit à réagir et à opérer une décision stratégique. Ces virages stratégiques changent la nature des activités et nous semblent être les points de rupture marquant la fin d'un cycle, pour en commencer un nouveau. Les différentes étapes et les points de rupture seront exposés de manière plus approfondie dans la présentation des résultats du cas $\mathrm{H} 2 \mathrm{H}$. 


\subsection{Présentation du cas de la TPE touristique $\mathrm{H} 2 \mathrm{H}$}

Filière dynamique et en perpétuelle métamorphose, le tourisme connaît depuis une dizaine d'années de profondes transformations issues, en partie, du développement de nouveaux systèmes de diffusion de l'information. Si l'adéquation des TIC au secteur du tourisme a si bien réussi, c'est que l'information se trouve au cœur du secteur touristique (Sheldon, 1993; Werthner et Klein, 2004). En fait, le processus de production du produit touristique met en œuvre un ensemble d'entreprises plus ou moins indépendantes, plus ou moins proches géographiquement, intervenant de façon plus ou moins rapprochée dans le temps, de nature souvent différente (entreprises marchandes et institutions publiques) et de tailles distinctes (artisanales et désorganisées pour les unes et largement industrialisées pour les autres). Elles interviennent de manière complémentaire afin de proposer un bien homogène au client (Eadington et Redman, 1991). Les entreprises coopèrent de plus en plus indirectement dans la réalisation d'un produit final qu'elles ne maîtrisent pas entièrement. L'échange d'informations (concernant les prix, les réservations, les disponibilités, les dates et horaires, etc.) est alors au centre de ce mécanisme de coordination et requiert des systèmes de gestion et de diffusion de l'information toujours plus innovants. Ces technologies ont émergé pour répondre précisément à ce besoin croissant de coordination et de traitement de l'information. L'évolution de la filière touristique est autant due à l'apparition de nouvelles technologies qu'aux changements qu'entraîne leur diffusion dans la façon de produire, de consommer et d'organiser le travail. Ainsi, la filière touristique s'est vite reconfigurée autour de l'utilisation de ces TIC, entraînant l'arrivée de nouveaux entrants, de mouvements de concentration, ainsi que de nouvelles coopérations sur le marché. Ces évolutions industrielles ont conduit les opérateurs à des comportements coopétitifs (penser sa stratégie en combinant à la fois des rapports de coopération et de concurrence).

Dans ce contexte, l'étude de la TPE touristique $\mathrm{H} 2 \mathrm{H}$ nous est apparue comme un terrain d'investigations privilégiées pour l'analyse du processus d'innovation et pour repérer des compétences nécessaires à sa réalisation. Il s'agissait, en outre, d'étudier un terrain jusqu'alors très peu exploré, puisque nous avons opté plus particulièrement pour le domaine de la réservation hôtelière et le traitement de l'information.

$\mathrm{H} 2 \mathrm{H}$ est à la fois le nom de l'entreprise que nous avons étudiée et le nom du système innovant promu par les deux collaborateurs dirigeants de l'entreprise. D'une part, H2H est une SARL (société à responsabilité limitée) niçoise au capital de 10000 euros, créée en octobre 2003 par trois associés (deux porteurs du projet et un apporteur de fonds). Les deux membres actifs de cette TPE sont madame Rita M., gérante et propriétaire de deux 
hôtels à Nice, et de monsieur Jacques T., qui a travaillé pendant 15 ans dans une centrale de réservation. Alors que madame M., en plus de son activité hôtelière, s'occupe de la gestion courante de $\mathrm{H} 2 \mathrm{H}$, monsieur T. se concentre, quant à lui, sur le développement du produit et sa commercialisation. À la création de la société, ils n'ont aucun employé. Bien que chacun soit spécialisé dans une tâche, les décisions importantes (stratégiques, financières, etc.) sont prises en commun. La coordination se fait par ajustement mutuel (les deux gérants travaillent dans le même bureau dans l'un des deux hôtels de madame M.) et la formalisation y est très faible.

D'autre part, H2H est également le nom du système original de réservations hôtelières. En effet, le principe est le suivant: en cas de surcapacité, les hôteliers qui effectuent les réservations proposent leur surplus de clients (loisirs et affaires) aux autres membres du réseau grâce à un Extranet ${ }^{5}$ les reliant en permanence les uns aux autres. Ainsi, les hôteliers en sous-capacité peuvent bénéficier des clients des hôtels complets ${ }^{6}$. C'est ainsi que $\mathrm{H} 2 \mathrm{H}$ est une TPE qui a pour objet de fédérer un réseau de PME touristiques indépendantes, sur la destination touristique de la Côte d'Azur.

De plus, cet outil calcule automatiquement la répartition des commissions entre partenaires et permet de réduire au maximum les coûts de ce type de service: pas de frais d'inscription, disparition d'intermédiaires et pratique du yield management ${ }^{7}$. La logique du système s'exprime de la manière suivante: l'attractivité de l'offre dépend de l'étendue du réseau (infrastructure et nombre de clients déjà connectés). Plus les hôteliers sont nombreux à appartenir au réseau $\mathrm{H} 2 \mathrm{H}$, plus de nouveaux hôtels sont incités à le rejoindre. C'est ainsi que $\mathrm{H} 2 \mathrm{H}$ bénéficie de la loi des rendements croissants d'adoption, dans la mesure où chaque nouvel adhérent le rend plus attractif pour les autres hôteliers. Partant de ce constat, chacun voulait attendre que le réseau s'étoffe pour y entrer. Dès les premières réservations effectuées, il a fallu réajuster le programme à la réalité. Les hôteliers ont participé à

5. L'Extranet élargit l'accès au réseau Intranet à un public extérieur à l'entreprise, public restreint et sélectionné (clients, fournisseurs).

6. À titre de précision, il existe deux formes de chaînes hôtelières: intégrées et volontaires. Une chaîne hôtelière intégrée est un groupe d'entreprises soumis à une unité de direction. Les chaînes hôtelières volontaires sont des regroupements d'entreprises indépendantes qui, sous des formes diverses, mettent leurs moyens en commun. $\mathrm{H} 2 \mathrm{H}$ est plutôt une forme de réseau volontaire d'entreprises.

7. Le yield management (désigné également sous le terme de «revenue management » ou encore de «tarification en temps réel») «est une technique qui permet de calculer, en temps réel, les meilleurs prix pour optimiser le profit généré par la vente d'un produit ou d'un service, sur la base d'une modélisation et d'une prévision en temps réel du comportement de la demande par microsegment de marché» (Site d'Amadeus, <http://www.optims.com/hight_profits.html>). 
cette évolution. $\mathrm{H} 2 \mathrm{H}$ est un produit fini mais qui continue d'évoluer. Les améliorations apportées permettent au réseau de s'étendre à tous les hôtels, du non-classé au trois-étoiles sur le territoire azuréen, quels que soient leur navigateur et l'installation informatique.

Cette innovation $\mathrm{H} 2 \mathrm{H}$ permet également au client qui trouverait un hôtel complet d'être immédiatement informé sur d'autres possibilités d'hébergement et, donc, de le fidéliser. Le partage des informations est instantané, et jour et nuit. La qualité des relations clients-hôteliers est optimisée.

En définitive, la raison d'être du projet $\mathrm{H} 2 \mathrm{H}$ est de réorganiser un système, existant mais insatisfaisant. Il propose un mode de fonctionnement bénéfique pour tous, clients et hôteliers, dans un état d'esprit d'entraide au sein d'une corporation, tout en réduisant une concurrence exacerbée. Ce programme a pour but de répartir de façon plus harmonieuse la rente touristique. L'idée est de canaliser les clients dans un cercle d'hôteliers pour la promotion d'un territoire touristique (une ville, une destination ou une région). $\mathrm{H} 2 \mathrm{H}$ se concentre actuellement sur la création d'un réseau d'hôteliers basés principalement sur la Côte d'Azur, mais les associés comptent développer et étendre le réseau à toute la France dans un axe futur de développement.

\section{Principaux résultats}

Dans cette partie, nous apportons des éléments de réponse à nos deux questions de recherche, que nous rappelons ici: Comment se réalise un processus d'innovation dans une TPE? Quelles sont les compétences nécessaires pour sa réussite? C'est ainsi que dans un premier temps (3.1), nous décrirons le processus d'innovation $\mathrm{H} 2 \mathrm{H}$ dans la TPE touristique du même nom que le système innovant. Dans un second temps (3.2), l'analyse empirique nous permettra de mettre en évidence les principales compétences mises en œuvre dans le processus d'innovation $\mathrm{H} 2 \mathrm{H}$.

\subsection{Présentation des phases du processus de l'innovation $\mathrm{H} 2 \mathrm{H}$}

Notre analyse des données recueillies au sein du cas $\mathrm{H} 2 \mathrm{H}$ révèle l'existence de six phases et de cinq points de rupture, que nous proposons de décrire tour à tour.

La phase de découverte. Cette phase a débuté en 2001, avec la rencontre entre madame Rita M., gérante et propriétaire de deux hôtels à Nice et de monsieur Jacques T., fort de ses 15 années d'expériences dans une centrale 
de réservation. Après quelques collaborations professionnelles ${ }^{8}$, ils ont pu observer l'existence d'une inégalité de pouvoir entre centrales de réservation et hôteliers. En effet, les entreprises de petites tailles sont souvent dans l'incapacité de s'approprier la totalité de la création de valeur de leur activité, cette dernière étant alors captée par les centrales de réservation. Cette phase, qui a duré près d'un an et demi, s'apparente à la perception par les deux principaux acteurs d'une imperfection sur le marché. Cette dernière se traduit alors par une externalité négative, c'est-à-dire que les centrales de réservations font supporter un coût (manque à gagner) aux hôteliers en l'absence d'un marché de droits de propriété, grâce auquel les hôteliers lésés pourraient se faire payer par ceux qui les lèsent. La prise de conscience de cette imperfection constitue le point de rupture qui conduit à la phase de maturation de l'idée.

La phase de maturation. Elle a consisté à comprendre les sources du problème et à faire émerger une idée pouvant remédier à l'imperfection du marché. Assimilée à de la recherche appliquée, cette phase consiste à explorer les différentes pistes envisageables pour la résolution du problème. À l'issue de cette phase de réflexion, courant de l'année 2003, les acteurs se décident à mettre sur le marché un système informatisé permettant aux hôteliers de capter une part de revenu supplémentaire lorsqu'ils sont complets. Ils sont désormais conscients d'être freinés, voire bloqués par leur manque de compétences techniques. Ils reconnaissent dès lors la nécessité d'acquérir de nouvelles compétences complémentaires, notamment informatiques. Pour ce faire, ils décident de faire appel à un programmeur informatique. Ce point de rupture marque l'entrée dans la troisième phase.

La phase de développement. Cette phase se différencie de la précédente par la confiance grandissante que témoignent les acteurs quant à la faisabilité du projet et par la collaboration avec un nouveau partenaire technique dans la conception du projet. Chacun, fort de sa propre expérience, a permis au système d'évoluer et de prendre forme jusqu'à l'obtention d'un prototype. Connaissant parfaitement les professionnels à qui ils destinent le système, les créateurs savent qu'il faut créer un produit simple d'utilisation, nécessitant peu de temps d'apprentissage et compatible avec les nombreux systèmes de réservation déjà existants. Pour la réalisation d'un tel système, les trois collaborateurs ont travaillé par essai-erreur et par la réalisation de tests, commencés en interne au milieu de l'année 2003. Chaque situation que pouvait rencontrer un hôtelier devait être envisagée et simulée et chaque problème

8. À titre d'illustrations, monsieur T. aidait parfois madame M. à remplir son hôtel, en échange du versement d'une commission. Inversement, lorsque son hôtel était complet, madame M. proposait à monsieur T. son surplus de clients sans contrepartie. Monsieur T. redirigeait alors les clients supplémentaires de madame M. vers d'autres hôtels qui lui reversaient une commission. 
devait trouver une solution simple et rapide. Le produit créé consiste en une technologie sur mesure, telle qu'elle est présentée dans le point 2.2. Cette phase de développement marque une accélération dans le processus, comparée aux deux premières phases de conceptualisation et de recherche. En particulier, c'est la volonté des deux acteurs d'officialiser, institutionnellement, leur relation et le projet, qui les engage dans la phase de création d'une entreprise. Cette phase présente une rupture avec les précédentes, dans la mesure où elle marque un tournant en passant de la créativité du système à sa formalisation.

La phase de "création d'une entité». À la suite de la phase de développement du système $\mathrm{H} 2 \mathrm{H}$, les collaborateurs ressentent le besoin d'obtenir une reconnaissance institutionnelle et effectuent toutes les démarches administratives conséquentes (ouvrir un compte en banque, enregistrement de l'entreprise, etc.). C'est ainsi que le 16 octobre 2003, les collaborateurs créent la SARL H2H, du même nom que le système informatisé. Lors de la création de l'entreprise, l'équipe de $\mathrm{H} 2 \mathrm{H}$ se compose de trois associés: Rita M. (responsable administratif, comptabilité et communication), Jacques T. (responsable commercial) et Joseph K. (apporteur de fonds). Limités par leurs ressources humaines et financières, les deux porteurs du projet (Rita M. et Jacques T.) ont décidé d'installer le siège social de H2H dans l'un des hôtels de madame M. L'aide d'un avocat, maître Stéphane Z., a été précieuse dans l'établissement de documents légaux comme l'élaboration des contrats pour les futurs adhérents, des courriers de propositions et de confirmations des réservations. Dans la lignée de la création de l'entreprise, $\mathrm{H} 2 \mathrm{H}$ a déposé une enveloppe Soleau à l'INPI (Institut national de la propriété intellectuelle) fin 2003, afin de protéger le système de rétribution des commissions entre hôtels. Une fois l'ensemble des tests effectués ainsi que la création de l'entreprise $\mathrm{H} 2 \mathrm{H}$ réalisée, les associés se sont attelés à la fabrication du produit $\mathrm{H} 2 \mathrm{H}$.

La phase de production. Cette étape visait la fabrication du produit pour une future commercialisation. La fixation du prix du système a été particulièrement compliquée étant donné la difficulté à établir la liste des composantes du produit tangible et leur coût respectif. Ainsi, le montant de la commission pour $\mathrm{H} 2 \mathrm{H}$ a été déterminé en fonction du seuil de rentabilité que devait atteindre le système, le nombre minimal de clients nécessaires pour générer un bénéfice minimal, la marge commerciale compte tenu de la marge bénéficiaire souhaitée, des frais fixes et variables, des taxes et, enfin, de la période de l'année considérée. Le processus de l'innovation $\mathrm{H} 2 \mathrm{H}$ est

9. L'enveloppe Soleau, du nom de son inventeur, est une preuve d'antériorité d'une invention que l'on peut utiliser en France pour obtenir la date de certaines inventions ou d'une idée. 
marqué ici par un point de rupture, que nous pouvons qualifier de «normal», eu égard aux travaux de Poole (1983), à savoir le passage vers la commercialisation du système.

La phase de mise sur le marché. La phase de commercialisation démarre en février 2004. Le début d'activité a été particulièrement difficile du fait de la réticence de certains hôteliers à utiliser le système. En effet, ces derniers considéraient, d'une part, que la prime récupérée par $\mathrm{H} 2 \mathrm{H}(40 \%$ de la commission) était trop élevée et, d'autre part, ils étaient sceptiques quant à la gratuité d'adhésion au système. Les deux associés ont alors mis en œuvre différentes démarches marketing, facilitant la compréhension et la commercialisation du système $\mathrm{H} 2 \mathrm{H}$ (porte-à-porte, télémarketing, site Web avec de nombreux exemples, mode d'emploi disponible, formation téléphonique aux adhérents du réseau, etc.). Jacques T. est allé rencontrer directement et physiquement les hôteliers pour leur expliquer le concept du produit et leur montrer en temps réel comment il fonctionnait. Le réseau comptait au 25 février 2005, 203 établissements sur la Côte d'Azur et, en octobre 2006, plus de 440 membres sur toute la France.

Dans le schéma 2, nous proposons une représentation, synthèse de notre analyse processuelle par les phases. L'intérêt de cette schématisation est de faire ressortir les différentes phases et les points de rupture du processus d'innovation $\mathrm{H} 2 \mathrm{H}$.

\section{SCHÉMA 2}

\section{Le processus d'innovation $\mathrm{H}_{2} \mathrm{H}^{*}$}

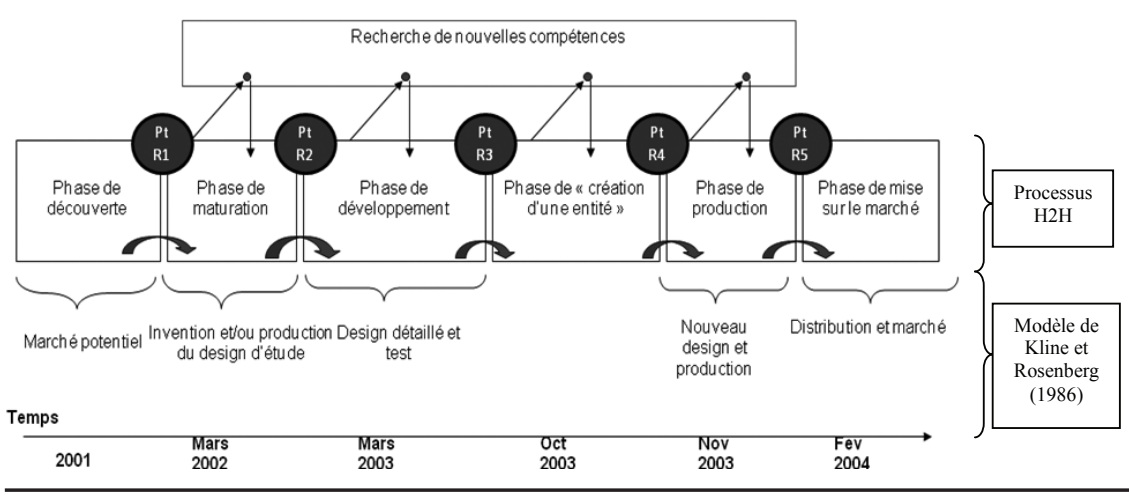

* Sur le schéma 2, les points R1 (imperfection de marché), R2 (manque de ressources techniques), R3 (volonté d'institutionnalisation), R4 (réalisation du produit) et R5 (lancement commercial du produit) représentent les points de rupture. 


\subsection{Mise en évidence des compétences pour innover}

Une fois analysées les phases du processus d'innovation $\mathrm{H} 2 \mathrm{H}$, il nous reste à traiter la question de la nature des compétences mises en œuvre dans le passage d'une phase à une autre. Concrètement, cette spécification s'est réalisée en analysant l'origine des points de rupture, telle qu'elle est expliquée dans la partie méthodologique ( $c f$. point 2.1). Cette analyse nous permet de dégager trois compétences majeures requises pour avancer dans le processus d'innovation: technologiques, organisationnelles et environnementales.

\subsubsection{Les compétences technologiques}

Madame M. a été précurseure en matière d'équipement technologique de ses établissements. En effet, elle décide de moderniser l'établissement qu'elle a racheté en 1992, lequel fonctionnait sans télécopieur, ni informatique. Puis en 1996, elle décide de s'équiper plus intensément:

Deux priorités se sont imposées: restaurer l'image de l'hôtel qui avait été négligée et trouver des débouchés, de nouvelles stratégies pour développer mon réseau clientèle (entretien de madame M., Azur Entreprises, novembre 2002).

À l'époque, Internet, alors à ses balbutiements dans le secteur touristique, lui apparaît comme un moyen de se démarquer.

J'ai été démarchée pour faire l'essai d'Internet au début. Très peu d'hôtels, surtout de petites tailles, en étaient équipés. Le premier site, qu'on a réalisé pour moi, était vraiment rudimentaire avec quelques photos et quelques lignes de texte. C'était un site vitrine, présentant la situation de l'hôtel et ses coordonnées pour un contact téléphonique, par télécopieur ou par courriel (entretien avec madame M., octobre 2004).

Malgré quelques résultats décevants (juste $5 \%$ de retombées), elle persévère en améliorant le graphisme de la page d'accueil et crée une base de données permettant aux clients potentiels de rentrer leurs dates de séjours et leurs coordonnées. Les réservations font un bond de $35 \%$ avec une augmentation du nombre de séjours étrangers. Autodidacte en informatique, madame M. a dû suivre des stages de formation. Ainsi, l'intérêt qu'éprouve la codirectrice de $\mathrm{H} 2 \mathrm{H}$ pour la technologie est avant tout un intérêt stratégique:

L'important, c'est de se faire connaître sur le Net. J'ai donc appris sur le terrain à me faire référencer et à consulter les moteurs de recherche (entretien avec madame M., octobre 2004).

Plus que l'augmentation de ses résultats, madame M. a gagné en crédibilité, ce qui lui a valu la confiance des banques. Dès lors, cet acteur a été 
un précurseur dans l'équipement et l'utilisation des nouvelles technologies de ses établissements. Avec une vision très claire de leur utilité et de leur usage, ses raisons d'utilisation sont essentiellement stratégiques et pratiques.

\subsubsection{Les compétences organisationnelles}

Les compétences organisationnelles sont intimement liées aux processus de management et aux éléments organisationnels construits autour de la connaissance et des savoir-faire individuels (Rouby et Thomas, 2004). Dans le cas $\mathrm{H} 2 \mathrm{H}$, nous avons repéré cinq principales compétences organisationnelles.

Un travail collaboratif par la mise en commun de ressources et de compétences complémentaires. La collaboration entre madame M. et monsieur T. est d'abord motivée par des intérêts communs visant à pallier le manque de ressources financières et humaines de chacun d'eux. En effet, ils exploitaient déjà au maximum leurs ressources pour leur activité initiale (l'hôtellerie pour madame $\mathrm{M}$. et les systèmes de réservation pour monsieur $\mathrm{T}$.) La saturation de leurs ressources les a naturellement conduits à collaborer. L'objectif prioritaire de la collaboration était le partage de ressources et de compétences complémentaires nécessaires à la création du projet $\mathrm{H} 2 \mathrm{H}$. Chacun des partenaires n'avait pas le contrôle total de l'action d'innover. Ce mode de travail collaboratif a été choisi dans le but de créer une valeur supérieure à la somme des valeurs de chacune des parties. Elle repose sur des synergies issues en partie du croisement de connaissances complémentaires. Les divers rapprochements qui ont eu lieu tout au long du processus d'innovation $\mathrm{H} 2 \mathrm{H}$ (rapprochement entre compétences hôtelières et compétences des réservations puis compétences techniques et, enfin, compétences juridiques) reposent sur la recherche de compétences complémentaires bien plus que sur la recherche d'une taille critique. En effet, le volume d'activités attendu dans les premières phases de commercialisation ne permettait pas d'envisager une expansion trop rapide des activités. C'est pour cette raison que les deux acteurs se sont essentiellement appuyés sur la coopération pour acquérir les compétences complémentaires à la réalisation de l'activité visée. Les acteurs ont su mobiliser les experts et développer une technologie sur mesure en faisant appel aux compétences techniques et fonctionnelles nécessaires. L'innovation n'est donc pas l'affaire d'un seul individu mais une affaire d'équipe: elle est un processus collectif. Les conditions de création de l'innovation reposent sur trois principes fédérateurs: la volonté de coopérer et l'interdépendance des ressources, les moyens de communication mis en place entre les acteurs et l'autonomie de chacun.

Une organisation par projet. Dans la littérature, il est reconnu que l'organisation par projet est propice à l'innovation (Gann et Salter, 2000). Dans 
le cas $\mathrm{H} 2 \mathrm{H}$ et comme expliqué ci-avant, il est intéressant de souligner que son organisation est en mode projet, dans la mesure où le projet de l'entreprise est le lancement d'un nouveau produit. En outre, le projet est porté par une équipe d'individus dotés de compétences diversifiées mobilisant plusieurs compétences fonctionnelles (marketing, comptabilité, production, etc.) (Loufrani-Fedida, 2007). Ici, les compétences sont fondamentalement liées à l'expérience accumulée dans le temps par les deux associés et confèrent ainsi un différentiel fonctionnel à l'entreprise.

Un apprentissage par l'expérience. Bien que n'ayant aucune formation spécifique, c'est l'expérience qui a conduit les deux associés à réaliser l'innovation. Nous avons observé que les associés ont une capacité d'apprentissage importante. Le monde hôtelier est soumis à une intense concurrence qui implique, pour les entreprises, de réaliser des «bonds en avant» nécessitant rapidité et savoir-faire. Les capacités d'apprentissage et de remise en cause des deux associés peuvent être considérées comme des compétences de l'entreprise.

Une coordination par la confiance. La collaboration entre les deux principaux acteurs s'est réalisée sur la base de la confiance. Selon Puthod (1995), la confiance est le fait de présumer, dans un accord, que chacune des parties va se comporter de façon conforme aux intérêts mutuels. Partant de ce principe, la formalisation du contrat liant les deux parties (indiquant la répartition des tâches et les répartitions financières) a été secondaire. Dans le cas $\mathrm{H} 2 \mathrm{H}$, nous avons observé une absence de formalisme ${ }^{10}$ qui est liée au fait que les protagonistes ont instauré une relation amicale et affective. Il est ainsi généralement admis que le relationnel, l'affect et l'intuition caractérisent fortement la stratégie des petites entreprises. Dans le cas $\mathrm{H} 2 \mathrm{H}$, une particularité surprenante apparaît concernant cette coordination par la confiance: le contrat a été refusé car il serait considéré comme une rupture à la confiance mutuelle et impliquerait une détérioration de la relation. Paradoxalement, il pourrait conduire à une méfiance du partenaire, le poussant ainsi à adopter un comportement opportuniste. Autant la confiance est perçue comme une compétence nécessaire à la collaboration, autant la contractualisation peut être perçue, à l'inverse, comme une forme de trahison.

Une culture de l'entrepreneuriat. Un risque d'échec dans le cas des collaborations réside dans la domination progressive d'un des acteurs sur son

10. Les acteurs ont seulement effectué des prises de notes et brouillons de leurs principales idées et de l'ensemble des tâches à effectuer. Ainsi, la plupart des connaissances des porteurs du projet $\mathrm{H} 2 \mathrm{H}$ sont tacites. Elles regroupent principalement les compétences innées et acquises des deux acteurs ainsi que leurs savoir-faire et expériences. 
partenaire. La volonté de prise de pouvoir par l'un des associés peut aboutir, soit à la rupture, soit à un rapport dominant-dominé non efficient. Dans le cas $\mathrm{H} 2 \mathrm{H}$, une condition favorable à la collaboration est la taille comparable des deux associés. Comme l'indique Saporta (1997), puisque le dirigeant est motivé au premier chef par des objectifs personnels, son entreprise est principalement destinée à lui permettre de les satisfaire. Par ailleurs, un trait de caractère des dirigeants s'est révélé marquant: les défis et la prise de risque ne leur font pas peur.

Relever le défi et intégrer la notion de risque, c'est-à-dire en avoir conscience mais qu'elle ne soit pas paralysante. Certains s'embourbent dans des calculs prévisionnels et ils en font tellement qu'ils ne créeront jamais. En effet, il faut à un moment donné arriver à se jeter à l'eau (entretien avec madame M., décembre 2004).

\subsubsection{Les compétences environnementales}

Selon les termes de Retour (2005), les compétences environnementales font référence aux compétences détenues par des entités ou des acteurs hors du contrôle direct de l'entreprise, à savoir les fournisseurs, les clients, les laboratoires de recherche, les partenaires institutionnels et tous acteurs dont l'action peut influer sur l'entreprise ou le groupe. En d'autres termes, il s'agit de la capacité que peut avoir une entreprise à mobiliser des compétences dont elle n'a pas la maîtrise et qui se trouvent dans son environnement proche. Sousjacent à ce niveau d'analyse de la compétence en émergence, nous trouvons la question classique des choix d'impartition de l'entreprise, symbolisée par la fameuse question: faire ou faire faire? Il s'agit ainsi de déterminer quelles sont les compétences qui seront mobilisées en interne et celles qui seront mises en œuvre à l'extérieur de l'entreprise. Ce niveau d'analyse commence à être étudié par les chercheurs en gestion des ressources humaines (GRH) et en stratégie dans le cadre des stratégies de coopération. En effet, levier de création et de transfert de connaissances, la coopération permet l'accès aux compétences du partenaire afin de faire émerger de nouvelles compétences communes (Quélin, 1997).

Dans le cas $\mathrm{H} 2 \mathrm{H}$, nous constatons que l'entreprise interagit de manière efficiente avec d'autres entrepreneurs (un avocat, un programmeur, etc.), afin de bénéficier de leurs compétences et de réduire les obstacles liés à sa petite taille. Les relations que la TPE entretient avec ses différents partenaires, notamment les fournisseurs spécialisés et son insertion dans des réseaux semblent déterminer sa capacité à acquérir des R-C manquantes, dénommée 
capacité d'absorption au sens de Cohen et Levinthal (1990) ${ }^{11}$. De fait, la capacité d'innovation de l'entreprise $\mathrm{H} 2 \mathrm{H}$ se construit, d'une part, à partir de son aptitude à acquérir de l'information dans le cadre des interactions avec son environnement et, d'autre part, de sa capacité à intégrer et traiter cette information, dans le cadre d'interactions internes, afin de générer de nouvelles connaissances. Aussi, c'est la reconnaissance de leur manque de compétences qui pousse les acteurs à rechercher, à l'extérieur, les compétences nécessaires pour avancer dans le processus d'innovation.

En synthèse, nous proposons, ci-après, un tableau récapitulant les compétences à l'œuvre lors du processus d'innovation $\mathrm{H} 2 \mathrm{H}$ ( $c f$. tableau 1).

\section{TABLEAU 1}

\section{Les compétences mises en ouvre lors du processus d'innovation dans la TPE touristique $\mathrm{H2H}$}

\section{Compétences technologiques}

Utilisateurs avertis et curieux des TIC, bonne maîtrise des outils.

Les outils doivent répondre à la capacité stratégique des entreprises (réduction des coûts, accès à de nouveaux marchés, différenciation, gains de productivité) sans investissement supplémentaire en termes de ressources et de compétences.

Compétences organisationnelles

Travail collaboratif.

Organisation par projet.

Apprentissage par expérience.

Coordination par la confiance.

Culture de l'entrepreneuriat.

Volonté de la direction de se distinguer de la concurrence et d'affecter du temps et quelques ressources pour innover, appuyée par des employés impliqués.

Les dirigeants s'engagent clairement vis-à-vis de l'innovation.

Compétences environnementales

Capacité de décrypter l'environnement.

Volonté de créer un réseau d'entreprises.

Capacité à acquérir des R-C manquantes.

Capacité à traiter l'information.

11. Cohen et Levinthal (1990) définissent la notion de capacité d'absorption comme «la capacité d'une entreprise à reconnaître la valeur d'une information nouvelle, externe, de l'assimiler et de l'exploiter à des fins commerciales» (p. 128). 


\section{Discussion}

Lors de l'analyse du processus d'innovation $\mathrm{H} 2 \mathrm{H}$, il est apparu de fortes similitudes avec le modèle en chaîne de Kline et Rosenberg (1986), que nous avons largement explicité au point 1.2. Trois raisons majeures confortent cette affirmation.

Premièrement, leur modèle propose l'enchaînement de cinq phases principales (marché potentiel, invention et/ou production du design d'étude, design détaillé et test, nouveau design et production, distribution et marché). Dans le cas $\mathrm{H} 2 \mathrm{H}$, la chaîne centrale est composée de six phases qui s'enchaînent en partie linéairement. La différence tient à la phase de création de l'entreprise qui est un acte unique.

Deuxièmement, les différentes phases du cas $\mathrm{H} 2 \mathrm{H}$ ne sont pas totalement indépendantes, comme dans le modèle que proposent les auteurs. Elles s'imbriquent les unes aux autres. De plus, lorsque l'une n'était pas aboutie, il a souvent été difficile pour l'entreprise $\mathrm{H} 2 \mathrm{H}$ de passer à la suivante. Pour chacune des phases, les activités réalisées présentent un caractère progressif. Ainsi, cet aspect graduel nous amène à penser que les phases ne sont pas entièrement prédictibles et que l'innovation progresse par tâtonnement (ou essai-erreur-nouvelle tentative) et par une lente accumulation de connaissances les faisant parfois reculer pour trouver d'autres pistes d'avancement.

Troisièmement, notre cas révèle que les acteurs ont fait appel à la science et à la technique lorsque leurs propres connaissances étaient insuffisantes. C'est ainsi que nous confirmons les propos de Kline et Rosenberg (1986), quant à la mobilisation de la recherche lorsque les connaissances existantes ne sont pas suffisantes pour résoudre les problèmes auxquels les dirigeants doivent faire face.

Toutefois, outre ces similitudes du processus d'innovation $\mathrm{H} 2 \mathrm{H}$ avec le modèle en chaîne de Kline et Rosenberg (1986), notre analyse en termes de compétences nous a permis d'expliquer comment l'entreprise $\mathrm{H} 2 \mathrm{H}$ est passée d'une phase à une autre et pourquoi elle cherche dans son environnement les R-C qui lui manquent. En effet, lors de notre analyse empirique, nous avons constaté que de nombreuses difficultés (le manque de ressources, les réseaux déjà constitués et les barrières à l'entrée qu'ils occasionnent, les démarches administratives, les risques économiques perçus excessifs, les coûts de conception trop élevés, l'absence de personnel qualifié ou encore le poids des charges) auraient pu conduire les dirigeants de $\mathrm{H} 2 \mathrm{H}$ à ne pas continuer le processus d'innovation. Pourtant, ils ont su identifier les R-C qui leur manquaient et mettre en place les actions managériales (formation, 
partenariat, relations contractuelles, etc.) pour acquérir ces compétences. C'est en mobilisant ces dernières que les dirigeants ont pu contourner les difficultés précitées et donc avancer dans le processus d'innovation.

Au-delà de la reconnaissance du manque de compétences comme explication du passage d'une phase à une autre du processus d'innovation dans les TPE, notre recherche soulève également la question de la nature des compétences. À ce sujet, le dernier rapport de la Commission européenne sur l'innovation, en date de 2006, indique que les obstacles les plus difficiles à surmonter pour les innovateurs ne sont généralement pas d'ordre technique, sans toutefois préciser la nature exacte de ces obstacles. Or, notre analyse du cas $\mathrm{H} 2 \mathrm{H}$ nous a permis de dégager trois compétences majeures requises pour avancer dans le processus d'innovation: technologiques, organisationnelles et environnementales.

\section{Conclusion}

Cet article visait à améliorer la compréhension du processus d'innovation au sein des TPE en mettant l'accent sur deux axes: le processus d'innovation en tant que tel, d'une part, et les compétences nécessaires à sa réussite, d'autre part.

Tout d'abord, l'étude longitudinale réalisée a permis de révéler six phases caractéristiques de l'innovation considérée $\mathrm{H} 2 \mathrm{H}$ et d'identifier cinq points de rupture, marquant le passage entre les phases. Cette analyse offre une vérification empirique du modèle de Kline et Rosenberg (1986). Ensuite, le cas $\mathrm{H} 2 \mathrm{H}$ a permis de repérer l'existence de compétences favorables au développement de l'innovation dans une TPE, à savoir les compétences technologiques, organisationnelles et environnementales. Notre recherche offre ainsi un prolongement de la littérature existante s'interrogeant sur le processus d'innovation dans les PME/TPE (Chanal, 2002; Fort, Rastoin et Temri, 2005 ; Ayerbe, 2006; Boldrini, 2008), et ce, en proposant un repérage des compétences requises pour mieux comprendre le processus d'innovation.

Il convient également de souligner les contributions de notre recherche sur le plan managérial. En particulier, cet article fournit aux managers une description concrète et rigoureuse du processus d'innovation au sein d'une TPE touristique ainsi que des compétences (technologiques, organisationnelles et environnementales) sollicitées en interne et en externe pour avancer dans ce processus.

Au-delà des contributions théoriques et managériales de notre travail, des limites essentiellement méthodologiques sont à signaler. La première d'entre elles a trait à la validité externe des résultats énoncés et, donc, à la 
portée des résultats: les compétences pour innover en TPE sont-elles spécifiques? À ce stade de notre recherche, nous ne pouvons prétendre que nos résultats soient applicables de façon large. Toutefois, la volonté initiale de notre travail n'a pas été de tendre vers une généralisation statistique, mais vers une généralisation analytique (Yin, 1994), dans le but d'enrichir, nous le rappelons, les travaux les plus récents sur le processus d'innovation dans les TPE. À cette limite inhérente à toute étude de cas s'ajoute une limite qui relève plus spécifiquement de la période d'observation de six mois, qui peut sembler insuffisante a priori pour l'observation du phénomène étudié. Néanmoins, ce travail étant essentiellement exploratoire, notamment au niveau de l'analyse des compétences requises dans un processus d'innovation, il n'en demeure pas moins une première étape dans la compréhension du processus d'innovation dans les TPE.

Finalement, l'évocation des limites du présent travail nous amène à relever quelques pistes de recherches qu'il apparaît prometteur de poursuivre à l'avenir. Une première voie de recherche consisterait à étendre la démarche suivie à de nouveaux terrains d'investigation à d'autres entreprises touristiques afin d'en améliorer la validité externe, car nous sommes bien conscientes que nos résultats liés à une seule étude de cas bénéficient d'une validité interne forte, au détriment d'une validité externe. Une seconde voie consisterait à mieux comprendre comment se complètent et se combinent les trois compétences identifiées tout au long du processus d'innovation. Pour ce faire, il serait intéressant de retourner sur le terrain d'étude $\mathrm{H} 2 \mathrm{H}$, ce que nous envisageons de réaliser prochainement. Enfin, bien qu'écartée de la présente étude pour des contraintes de taille imposées de l'article, une troisième orientation de recherche résiderait dans l'analyse du niveau territorial des compétences. En effet, la compétence environnementale rassemble des acteurs travaillant à plusieurs centaines ou milliers de kilomètres les uns des autres; elle devient territoriale quand elle se réfère à une combinaison de ressources géographiquement proches, permettant au territoire d'afficher une spécialisation compétitive (Defélix et Mazzilli, 2009). Ce dernier point fera d'ailleurs l'objet d'un futur programme de recherche sur la gestion de la compétence territoriale de la destination touristique azuréenne.

En définitive, nous espérons que notre travail a contribué à une meilleure compréhension du processus d'innovation dans les TPE, et ce, grâce à la mobilisation du cadre théorique des $\mathrm{R}-\mathrm{C}$ permettant le repérage des compétences requises pour innover. Plus généralement, cette recherche se veut être un premier pas dans l'identification des compétences nécessaires au processus d'innovation dans les TPE. 


\section{Bibliographie}

AKrich, M., M. CALlon et B. LATOUR (1988), «À quoi tient le succès des innovations?», Gérer et comprendre, premier épisode: «L'art de l'intéressement», no 11, juin, p. 4-17; deuxième épisode : «L'art de choisir les bons porte-parole», $\mathrm{n}^{\circ} 12$, septembre, p. $14-29$.

AYERBE, C. (2006), «Innovations technologique et organisationnelle au sein de PME innovantes: complémentarité des processus, analyse comparative des mécanismes de diffusion », Revue internationale PME, vol. 19, n 1, p. 11-33.

BARNEY, J.B. (1991), «Firm resources and sustained competitive advantage », Journal of Management, vol. 17, n 1, p. 99-120.

BELlON, B. (1994), Innover ou disparaître, Paris, Économica.

BOLDRINI, J.-C. (2008), «Caractériser les pratiques de conception des PME pour mieux accompagner leurs projets d'innovation», Revue internationale PME, vol. 21, n 1 , p. 9-34.

BRÉCHET, J.-P. (1990), «Pour une analyse stratégique adaptée à la PMI», Revue française de gestion, juin-juillet-août, p. 19-24.

CHANAL, V. (2002), «Comment accompagner les PME-PMI dans leur processus d'innovation? », Actes de la XI Conférence de l'Association internationale de management stratégique, Paris, juin.

Cohen, W.M. et D.A. Levinthal (1990), «Absorptive capacity: a new perspective on learning and innovation », Administrative Science Quarterly, vol. 35, n 1 , p. $128-152$.

DECELLE, F.X. et J.F. TASSIN (2005), Tourisme et innovation: bilan et perspectives, La Documentation française, coll. «Conseil national du tourisme».

DefÉliX, C. et I. MAZZILLI (2009), «De l'individu au territoire: la longue marche de la gestion des compétences», dans D. Retour, T. Picq et C. Defélix (dir.), Gestion des compétences: nouvelles relations, nouvelles dimensions, Paris, Vuibert, p. 197-209.

EAdington, W.R. et M. Redman (1991), «Economics and tourism», Annals of Tourism Research, vol. 18, n 1, p. 41-56.

FORT, F., J.L. RASTOIN et L. TEMRI (2005), «Les déterminants de l'innovation dans les petites et moyennes entreprises agroalimentaires», Revue internationale $P M E$, vol. 18, no 1, p. 47-72.

François, J.P., D. GouX, D. Guellec, I. Kabla et P. TemPle (1999), «Décrire les compétences pour l'innovation: une proposition d'enquête», dans D. Foray et J. Mairesse (dir.), Innovations et performances: approches interdisciplinaires, Paris, Éditions de l'École des Hautes Études en sciences sociales, p. 283-303.

GANN, D. et A. SALTER (2000), «Innovation management in project-based, serviceenhanced firms : the construction of complex products and systems », Research Policy, vol. 29, p. 955-972. 
GRANT, R.M.(1991), «The resource-based theory of competitive advantage: implications for strategy formulation», California Management Review, vol. 33, n 3, p. $114-135$.

JAOUEN, A. et O. TORRÈS (2008), Les très petites entreprises: un management de proximité, Paris, Hermès Science-Lavoisier.

JULIEN, A. (1996), «Information control: a key factor in small business development», 41th International Congress on Small Business (ICSB), Stockholm, 17-19 juin.

KLINE, S.J. et N. RosenBERG (1986), «An overview of innovation », dans R. Landau et N. Rosenberg (dir.), The Positive Sum, Academy of Engineering Press, p. 275-305.

LOILIER, T. et A. Tellier (1999), Gestion de l'innovation: décider, mettre en ouvre, diffuser, Caen, Éditions Management \& Société.

LOUFRANI-FEDIDA, S. (2007), «Les compétences fonctionnelles et d'intégration: des compétences essentielles aux projets de développement de nouveaux produits. Le cas des compléments alimentaires », Revue française de gestion industrielle, $\mathrm{n}^{\circ} 2$, juin, p. 37-58.

MARChESNAY, M. (1997), «Petite entreprise et entrepreneur», dans Y. Simon et P. Joffre (dir.), Encyclopédie de Gestion, Tome 2, 2e édition, Paris, Économica, p. 2209-2219.

Mintzberg, H. (1982), Structure et dynamique des organisations, Paris, Éditions d'Organisation.

NANDA, A. (1996), «Resources, capabilities and competencies», dans B. Moigeon et A. Edmonson (dir), Organizational Learning and Competitive Advantage, Londres, Sage Publications, p. 93-120.

OsÉO (2006), «PME et innovation technologique: pour une relation plus naturelle», Regards sur les $P M E, \mathrm{n}^{\circ} 10$.

PoOLE, M.S. (1983), «Decision development in small groups: a multiple sequence model of group decision development », Communication Monographs, vol. 50, p. 321-340.

PRAHALAD, C.K. et G. HAMEL (1990), «The core competence of the corporation », Harvard Business Review, vol. 68, n 3, p. 79-91.

PUTHOD, D. (1995), Les alliances de PME: stratégie de développement et implications managériales, Thèse de doctorat en sciences de gestion, Université de Savoie.

QUÉLIN, B. (1997), «Appropriability and the creation of new capabilities through strategic alliances », dans R. Sanchez et A. Heene (dir.), Strategic Learning and Knowledge Management, New York, John Wiley \& Sons, p. 139-160.

RETOUR, D. (2005), «Le DRH de demain face au dossier compétences », Management et Avenir, no 4, avril, p. 187-200.

Rouby, E. et C. Thomas (2004), «La codification des compétences organisationnelles : l'épreuve des faits », Revue française de gestion, vol.30, n 149, mars-avril, p. 51-68. 
SAPORTA, B. (1997), «Stratégies des petites et moyennes entreprises », dans Y. Simon et P. Joffre (dir.), Encyclopédie de Gestion, Tome 3,2e édition, Paris, Économica, p. 3105-3128.

SHELDON, P.J.(1993), «Destination information systems », Annals of Tourism Research, vol. $20, n^{\circ} 4$, p. 633-649.

TARONDEAU, J.C. (2002), Le management des savoirs, 2e édition, n 3407, Paris, Presses universitaires de France, coll. «Que sais-je?».

TeEce, D.J., G. Pisano et A. Shuen (1997), «Dynamic capabilities and strategic management», Strategic Management Journal, vol. 18, n 7, p. 509-533.

TORRÈs, O. (1997), «Pour une approche contingente de la spécificité de la PME», Revue internationale PME, vol. 10, nº 2, p. 9-43.

TORRÈs, O. (2004), «The SME concept of Pierre-André Julien: an analysis in terms of proximity », Piccola Impresa, vol. 2, p. 51-61.

TORRÈs, O. (2007), «Approche descriptive de la spécificité de gestion des PME: le mix de proximité», dans L.J. Filion (dir.), Management des PME: de la création à la croissance, Saint-Laurent, Québec, Éditions du Renouveau pédagogique, p. 23-34.

VAN DE VEN, A.H. et M.S. PoOLE (1990), «Methods for studying innovation development in the Minnesota innovation research program», Organization Science, vol. 1, p. 313-335.

WERnERFELT, B. (1984), «A resource-based view of the firm », Strategic Management Journal, vol. 5, n 1 , p. 171-180.

WERTHNER, H. et S. KLEIN (2004), «Mobil challenges for travel and tourism », Conference on Information Systems, Europe.

YIN, R.K. (1994), Case Study Research:Design and Methods, $2^{\mathrm{e}}$ édition, Applied Social Research Methods Series, vol. 5, Londres, Sage Publications. 